\title{
Optical and Thermal Diffusivity Measurement of Ge-Se-Te Glasses by Photoacoustic Technique
}

\author{
R. Ganesan (a), K. N. Madhusoodanan (b), A. Srinivasan (c), \\ K. S. SAngunNi ${ }^{1}$ ) (a), and E. S. R. Gopal (a) \\ (a) Department of Physics, Indian Institute of Science, Bangalore - 560012, India \\ (b) Department of Instrumentation, Cochin University, Cochin, India \\ (c) Department of Physics, Indian Institute of Technology, Guwahati, India
}

\begin{abstract}
The composition dependence of optical energy gap $\left(E_{\mathrm{g}}\right)$ and thermal diffusivity $(\alpha)$ in three sets of $\mathrm{Ge}-\mathrm{Se}-\mathrm{Te}$ glasses, namely, $\mathrm{Ge}_{x} \mathrm{Se}_{80-x} \mathrm{Te}_{20} \quad(5 \leq x \leq 40), \mathrm{Ge}_{x} \mathrm{Se}_{75-x} \mathrm{Te}_{25} \quad(5 \leq x \leq 40)$ and $\mathrm{Ge}_{x} \mathrm{Se}_{65-x} \mathrm{Te}_{35}(5 \leq x \leq 37.5)$, have been studied using photoacoustic technique. The variation of $\alpha$ with average coordination number $\langle r\rangle$ shows a change in the slope at $\langle r\rangle=2.4$ and 2.67. $E_{\mathrm{g}}$ shows a local maximum at $\langle r\rangle=2.67$. The results are discussed on the basis of the topological and chemical threshold exhibited by covalent network glasses. The relative sensitivity of $\alpha$ and $E_{\mathrm{g}}$ to these phenomena is discussed.
\end{abstract}

\section{Introduction}

Chalcogenide glasses exhibit electrical and optical properties which make them useful for several potential applications. Specifically, the threshold and memory switching behavior and the infrared transmission of many of these glasses make them potential materials for use in memory devices and fiber optics [1, 2]. Multi-component glasses were found to be more useful for many of these applications [3, 4]. Because of this there has been a great deal of interest in recent years in understanding the composition dependent variations of physical properties in these glasses. These investigations have established that many of the physical properties show specific features like extremum, kink, change of slope etc. at certain compositions [5,6]. Models based on chemical ordering [7] and network topology [8 to 11] have been proposed to explain the composition dependence of physical properties. The chemically ordered network (CON) model favors the formation of heteropolar bonds and thus the glass structure is composed of crosslinked structural units of stable chemical compounds and excess, if any, of the elements. It has been argued that chemical ordering leads to a chemical threshold at which specific features in the composition dependent variations occur [12]. The topological model is based on balancing the number of operative constrains with the number of degrees of freedom. This model describes the composition dependence in terms of the average coordination number $\langle r\rangle$ and predicts a topological threshold at $\langle r\rangle=2.4$, where the rigidity of the network percolates [10]. The network is floppy below $\langle r\rangle=2.4$ and rigid above $\langle r\rangle=2.4$. A later modification of this model [11] based on the forma-

1) Corresponding author, e-mail: sangu@physics.iisc.ernet.in 
tion of two-dimensional layer structures and medium range interactions suggests a topological threshold at $\langle r\rangle=2.67$ where a change from two-dimensional layered structure to a three-dimensional network takes place due to cross linking.

The applicability of the ideas of rigidity percolation was verified in many binary and ternary glasses $[5,6,11,13]$. Signature of rigidity percolation has been reported to occur at 2.4 or near 2.67 in various glasses. Reports on the observation of threshold at 2.4 and 2.67 in glassy systems like $\mathrm{Ge}-\mathrm{Se}$ and $\mathrm{Ge}-\mathrm{As}-\mathrm{Se}$ have further complicated the issue. In binary glasses such as As-Se (V-VI glasses), the chemical stoichiometric composition occurs at $\langle r\rangle=2.4$ (the threshold proposed by Phillips and Thorpe [10]) and in Ge-Se glasses (IV-VI glasses) it occurs at $\langle r\rangle=2.67$ (which also happens to be the threshold value due to Tanaka [11]). A systematic study on the $\langle r\rangle$ dependence in ternary systems such as IV-V-VI glasses [14 to 18], As-Se-Te [19] and As-Sb-Se [20] was performed using photoacoustic spectroscopy, high-pressure resistivity and differential scanning calorimetry. These studies showed evidence for a threshold behavior at $\langle r\rangle=2.4$ for $\mathrm{As}-\mathrm{Se}-\mathrm{Te}$ and $\mathrm{As}-\mathrm{Sb}-\mathrm{Se}$ glasses. These results also seem to signify that the coordination of the various constituent atoms play a role in the observed threshold behavior. In the IV-V-VI glasses, the coordination (determined using the 8- $n$ rule) of the three elements are different whereas in V-VI-VI and V-V-VI glasses only two types of coordinations are involved. Hence it may be possible that, as far as threshold behavior is concerned, the V-VI-VI and V-V-VI glasses could behave as a binary glass. As already mentioned, many binary glasses show threshold at $\langle r\rangle=2.4$ [5]. Thus, the available experimental results suggest the need for further investigations on the composition dependence of physical properties in chalcogenide glasses.

In this paper, we present results on the composition dependence of thermal diffusivity and optical energy gap in three sets of Ge-Se-Te glasses, namely $\mathrm{Ge}_{x}-\mathrm{Se}_{80-x}-\mathrm{Te}_{20}$ $(x=5,10,15,20,25,30,33.33,35,40), \mathrm{Ge}_{x}-\mathrm{Se}_{75-x}-\mathrm{Te}_{25}(x=5,10,15,20,25,30$, $33.33,35,40)$ and $\mathrm{Ge}_{x}-\mathrm{Se}_{65-x}-\mathrm{Te}_{35}(x=5,10,15,20,25,30,33.33,35,37.5)$. The composition range covers the threshold composition predicted on the basis of various models. In terms of average coordination number $\langle r\rangle$, calculated using the formula

$$
\langle r\rangle=\frac{\left[(X) Z_{\mathrm{Ge}}+(Y) Z_{\mathrm{Te}}+(100-X-Y) Z_{\mathrm{Se}}\right]}{100},
$$

where $Z_{\mathrm{Ge}}=4, Z_{\mathrm{Te}}=2, Z_{\mathrm{Se}}=2$ are the coordination numbers of Ge, Te and Se, respectively, the compositions fall in the range $2.1 \leq\langle r\rangle \leq 2.8$.

\section{Experimental}

The glass-forming region for $\mathrm{Ge}-\mathrm{Se}-\mathrm{Te}$ consists of two islands [21]. The samples were prepared using the conventional melt-quenching technique. Appropriate amounts of high purity $(99.999 \%)$ constituent elements were sealed in a quartz ampoule at about $10^{-3} \mathrm{~Pa}$ and loaded in a rotary furnace. The ampoule was kept at $1000{ }^{\circ} \mathrm{C}$ for $12 \mathrm{~h}$ with a continuous rotation for homogenizing the mixture. It is then quenched in a mixture of $\mathrm{NaOH}$ and ice-water. The glassy nature of the sample was confirmed by X-ray diffraction and differential scanning calorimetry. The thermal diffusivity $(\alpha)$ and optical band gap $\left(E_{\mathrm{g}}\right)$ were measured using the photoacoustic technique. The description of photoacoustic spectrometer used and the details regarding the measurements of $\alpha$ and $E_{\mathrm{g}}$ were given elsewhere [22]. 


\section{Results and Discussion}

The variation of optical energy gap $E_{\mathrm{g}}$ as a function of average coordination number $\langle r\rangle$ for the three sets of glasses studied is given in Fig. 1. It can be inferred that the three sets of glasses show identical trends in the $\langle r\rangle$ dependence. $E_{\mathrm{g}}$ decreases initially as $\langle r\rangle$ is increased (i.e. when the Ge concentration is increased) and then exhibits a local maximum at $\langle r\rangle=2.67$. An explanation of the observed behavior can be given in the framework of the energy band model for chalcogenide glasses proposed by Kastner [23] and the change in the average bond energy of the system as the composition is varied. According to Kastner the valence band in chalcogenide glasses is constituted by the lone pair band whereas the conduction band arises from the anti-bonding band. In a multi-component glass like $\mathrm{Ge}-\mathrm{Se}-\mathrm{Te}$ the position of conduction and valence band edges and thus the energy gap largely depends on the relative number of various possible bonds in the system and the average bond energy. The various possible bonds in the $\mathrm{Ge}-\mathrm{Se}-\mathrm{Te}$ system are $\mathrm{Ge}-\mathrm{Se}, \mathrm{Se}-\mathrm{Se}, \mathrm{Se}-\mathrm{Te}, \mathrm{Ge}-\mathrm{Ge}, \mathrm{Ge}-\mathrm{Te}$, and $\mathrm{Te}-\mathrm{Te}$. The bond energies are 231.11, 189.22, 187.57, 205.57, 172.08, and $142.35 \mathrm{~kJ} / \mathrm{mol}$, respectively. The bond energies have been estimated by the method suggested by Pauling [24], from the bond energies of homopolar bonds [25] and the electronegativity values [26]. The CON model suggests that heteropolar bonds favored over homopolar bonds. Considering that the strong $\mathrm{Ge}-\mathrm{Se}$ bonds are energetically favored compared to a random mixture of bonding configurations, it is possible to consider the glasses studied as either chalcogen rich or Ge rich in the order of increasing $\langle r\rangle$. The observed initial decrease in $E_{\mathrm{g}}$ with increase in $\langle r\rangle$ suggests that in this region of the curve the influence of the relative number of $\mathrm{Se}-\mathrm{Se}$ and $\mathrm{Te}-\mathrm{Te}$ bonds are more prominent in determining the band gap than that of the $\mathrm{GeSe}_{2}$ structural unit. It appears that $\mathrm{Te}-\mathrm{Te}$ bonds are present in all these compositions. The decrease in $E_{\mathrm{g}}$ continues under the influence of the relative increase in the number of weak $\mathrm{Te}-\mathrm{Te}$ bonds, until the increase in Ge content

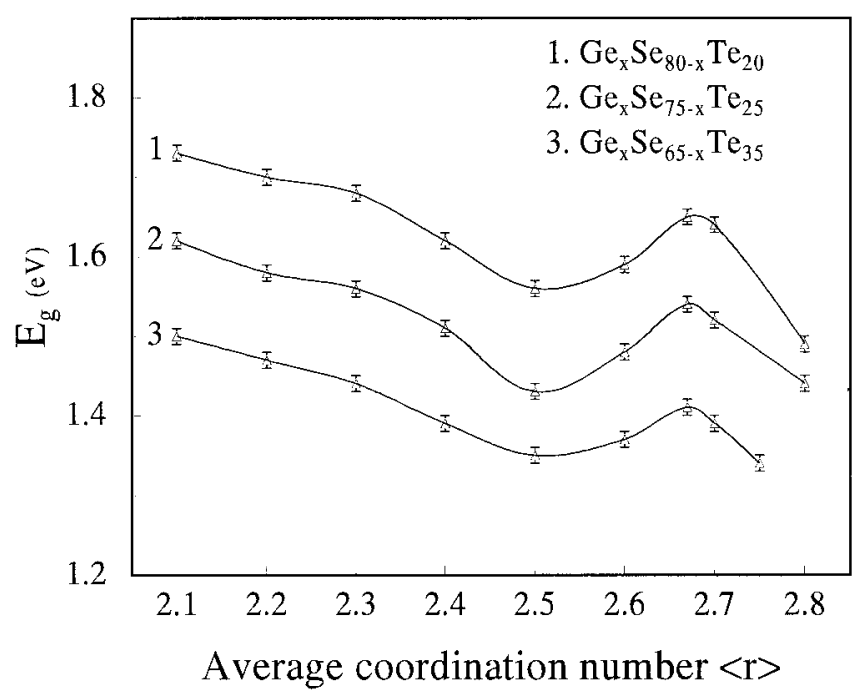

Fig. 1. Variation of optical energy gap $\left(E_{\mathrm{g}}\right)$ with average coordination number $\langle r\rangle$ for the three sets of $\mathrm{Ge}-\mathrm{Se}-\mathrm{Te}$ glasses 
is sufficient to produce Ge-Te bonds in the system. At $\langle r\rangle=2.67$, which corresponds to the $\mathrm{GeSe}_{2}-\mathrm{GeTe}_{2}$ pseudo-binary composition with possibly maximum average bond energy, $E_{\mathrm{g}}$ shows a maximum. At this composition the possibility for the formation of a maximum number of heteropolar bonds exists as compared to the homopolar bonds and consequently, a chemical ordering occurs in the glass. The composition with $\langle r\rangle>2.67$ contains a large concentration of relatively weak Ge-Ge bonds resulting in a decrease of $E_{\mathrm{g}}$. At this point it is interesting to note that $\mathrm{Ge}_{x} \mathrm{Se}_{100-x}$ shows a maximum in $E_{\mathrm{g}}$ at $\langle r\rangle=2.67$, which corresponds to the formation of stoichiometric $\mathrm{GeSe}_{2}$ glass [27]. The $\mathrm{Ge}_{x} \mathrm{Te}_{100-x}$ system also shows a similar trend [22]. The $E_{\mathrm{g}}$ variation in $\mathrm{Ge}-\mathrm{Se}-\mathrm{Te}$ does not show any specific feature at $\langle r\rangle=2.4$, the rigidity percolation threshold predicted by the topological model of Phillips and Thorpe [10]. Since $E_{\mathrm{g}}$ is more sensitive to the variation of the chemical bonding nature and the average bond energy, the local maximum occurring at $\langle r\rangle=2.67$ can be considered to be the signature of the chemical threshold. However, it may be mentioned that $\langle r\rangle=2.67$ also corresponds to the topological threshold suggested by Tanaka [11] based on the formation of a layered structure and medium range interactions.

The variation of thermal diffusivity $(\alpha)$ with $\langle r\rangle$ in $\mathrm{Ge}-\mathrm{Se}-\mathrm{Te}$ glasses is given Fig. 2 . It is observed that in all the three sets of glasses $\alpha$ increases as $\langle r\rangle$ increases, with a change of slope at $\langle r\rangle=2.4$. $\alpha$ increases more rapidly in the region $2.4\langle\langle r\rangle<2.67$ and there appears a saturation for $\langle r\rangle>2.67$. This behavior is consistent with the topological model for network glasses. The value of $\langle r\rangle=2.4$, at which the change of slope occurs, corresponds to the rigidity percolation threshold according to the topological model of Phillips and Thorpe [10]. The model divides the network into floppy and rigid with $\langle r\rangle=2.4$ as the critical value. Thermal diffusivity, which is directly related to thermal conductivity, depends on the phonon mean free path which in turn depends on the network structure. The zero frequency modes present in the floppy network (for which

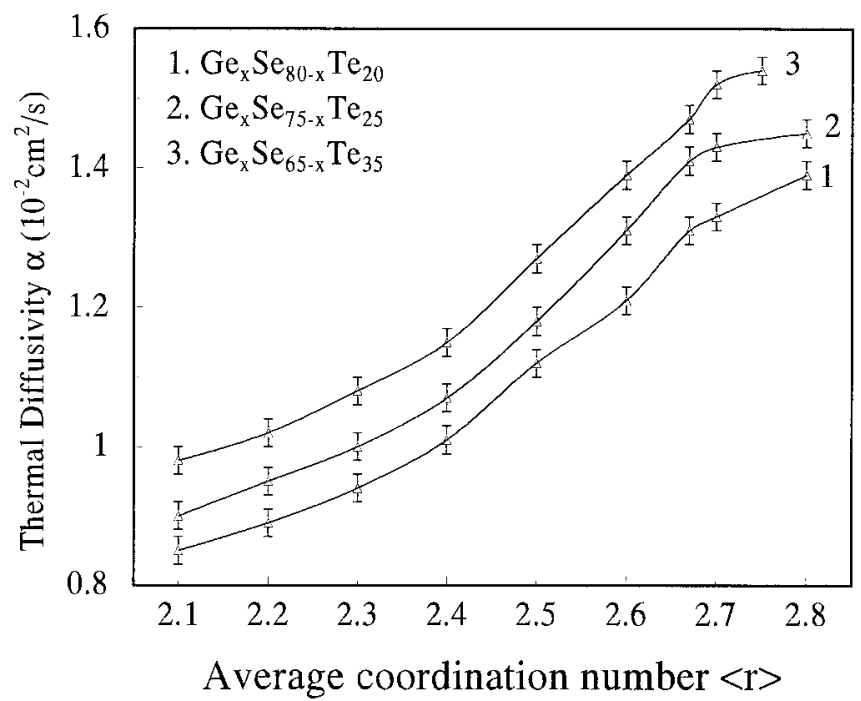

Fig. 2. Variation of thermal diffusivity $(\alpha)$ with a average coordination number $\langle r\rangle$ for the three sets of $\mathrm{Ge}-\mathrm{Se}-\mathrm{Te}$ glasses 
$\langle r\rangle<2.4)$ cause a scattering of thermal phonons, thus reducing the phonon mean free path. As $\langle r\rangle$ increases the number of zero frequency modes decreases and goes to zero at 2.4 when rigidity percolates in the network. This results in an increase in phonon mean free path as evidenced in the $\alpha$ versus $\langle r\rangle$ plot. Whether the saturation in $\alpha$ for $\langle r\rangle>2.67$, which corresponds to another topological threshold according to Tanaka, has got a topological origin is not very clear from the present results because this composition also corresponds to the chemical threshold.

\section{Conclusion}

The variation of $E_{\mathrm{g}}$ and $\alpha$ of the three sets of $\mathrm{Ge}-\mathrm{Se}-\mathrm{Te}$ glasses studied show features at different $\langle r\rangle$ values. These results can be interpreted as a signature of two different phenomena occurring in this glass system, namely, the chemical threshold and the topological threshold. Since $E_{\mathrm{g}}$ is more sensitive to variations of the relative number of different bonds and the average bond energy of the system the local maximum in the $E_{\mathrm{g}}$ variation is the signature of the chemical ordering occurring in the system at $\langle r\rangle=2.67$. The $\alpha$ data, being related to the phonon mean free path, on the other hand are more sensitive to the change in the network topology. Hence the feature exhibited in the $\alpha$ variation at around $\langle r\rangle=2.4$ should be an indication of a topological threshold. These results on the $\alpha$ variation support the topological model of Phillips and Thorpe for covalent glasses. Hence both chemical and topological thresholds exist in the $\mathrm{Ge}-\mathrm{Se}-\mathrm{Te}$ system and manifest in the properties that are sensitive to either of the phenomena.

Acknowledgements The authors thank Department of Science and Technology (DST) and Council of Scientific and Industrial Research (CSIR), Government of India, for the financial support of this work.

\section{References}

[1] D. AdLeR, Sci. Amer. 36, 236 (1977).

[2] J. A. Savage, P. J. Webber, and A. M. Pitt, IR Phys. 20, 313 (1980).

[3] H. Fritsche, in: Amorphous and Liquid Semiconductors, Ed. J. Tauc, Plenum Press, New York 1974 (p. 313).

[4] I. Haruvi-Busnach, J. Dror, and N. J. Croitoru, Mater. Res. 5, 1215 (1990).

[5] S. Asokan, G. Parthasarathy, and E. S. R. Gopal, Phil. Mag. B 57, 49 (1988).

[6] M. Tatsumisago, B. L. Halfpap, J. L. Green, S. M. Angell, and C. A. Angell, Phys. Rev. Lett. 64, 1549 (1990).

[7] G. Lucovsky and T. M. Hayes, in: Amorphous Semiconductors, Ed. M. H. Brodsky, SpringerVerlag, Berlin 1979 (p. 215).

[8] J. C. Phillips, J. Non-Cryst. Solids 34, 153 (1979).

[9] M. F. Thorpe, J. Non-Cryst. Solids 57, 355 (1983).

[10] J. C. Phillips and M. F. Thorpe, Solid State Commun. 53, 699 (1985).

[11] K. Tanaka, Phys. Rev. B 39, 1270 (1989).

[12] A. Giridhar, P. S. L. Narasimham, and S. Mahadevan, J. Non-Cryst. Solids 43, 29 (1981).

[13] A. Srinivasan, K. N. Madhusoodanan, and E. S. R. Gopal, Solid State Commun. 83, 163 (1992).

[14] A. Srinivasan, K. N. Madhusoodanan, E. S. R. Gopal, and J. Philip, Phil. Mag. B 65, 99 (1992).

[15] A. Srinivasan, K. N. Madhusoodanan, E. S. R. Gopal, and J. Philip, Phys. Rev. B 45, 8112 (1992). 
[16] A. Srinivasan, K. N. Madhusoodanan, E. S. R. Gopal, and J. Philip, J. Non-Cryst. Solids 155, 267 (1993).

[17] A. Srinivasan, K. Ramesh, K. N. Madhusoodanan, and E. S. R. Gopal, phys. stat. sol. (b) 170, K 83 (1992).

[18] A. SRinivasan, K. Ramesh, K. N. Madhusoodanan, and E. S. R. Gopal, Phil. Mag. Lett. 65, 249 (1992).

[19] K. NAndakumar and J. Philip, J. Non-Cryst. Solids 144, 247 (1992).

[20] K. Nandakumar and J. Philip, Phil. Mag. B 63, 493 (1991).

[21] Z. U. Borisova, Glassy Semiconductors, Plenum Press, New York 1981.

[22] K. N. Madhusoodanan, J. Philip, G. Parthasarathy, S. Asokan, and E. S. R. Gopal, Phil. Mag. B 58, 123 (1988).

[23] M. Kastner, Phys. Rev. Lett. 28, 355 (1972).

[24] L. Pauling, Nature of the Chemical Bonds, 1960 Cornell University Press, Ithaca (New York) 1960.

[25] R. T. Sanderson, Chemical Bonds and Bond Energy, 1976 Academic Press, New York 1976.

[26] A. L. Allred, in: Physical Chemistry Source Book, Ed. S. P. PArker, Mc Graw-Hill Publ. Co., New York 1987 (p. 186).

[27] K. N. Madhusoodanan and J. Philip, phys. stat. sol. (a) 108, 775 (1988). 